\title{
QUEEN'S
UNIVERSITY
BELFAST
}

\section{The impact of excluding or including Death Certificate Initiated (DCl) cases on estimated cancer survival: A simulation study}

Andersson, T. M-L., Myklebust, T. Å., Rutherford, M. J., Møller, B., Soerjomataram, I., Arnold, M., Bray, F., Parkin, D. M., Sasieni, P., Bucher, O., De, P., Engholm, G., Gavin, A., Little, A., Porter, G., Ramanakumar, A. V., Saint-Jacques, N., Walsh, P. M., Woods, R. R., \& Lambert, P. C. (2021). The impact of excluding or including Death Certificate Initiated (DCI) cases on estimated cancer survival: A simulation study. Cancer Epidemiology, 71(Pt A), 101881. https://doi.org/10.1016/j.canep.2020.101881

Published in:

Cancer Epidemiology

Document Version:

Peer reviewed version

Queen's University Belfast - Research Portal:

Link to publication record in Queen's University Belfast Research Portal

\section{Publisher rights}

Copyright 2021 Elsevier

This manuscript is distributed under a Creative Commons Attribution-NonCommercial-NoDerivs License

(https://creativecommons.org/licenses/by-nc-nd/4.0/), which permits distribution and reproduction for non-commercial purposes, provided the author and source are cited.

\section{General rights}

Copyright for the publications made accessible via the Queen's University Belfast Research Portal is retained by the author(s) and / or other copyright owners and it is a condition of accessing these publications that users recognise and abide by the legal requirements associated with these rights.

Take down policy

The Research Portal is Queen's institutional repository that provides access to Queen's research output. Every effort has been made to ensure that content in the Research Portal does not infringe any person's rights, or applicable UK laws. If you discover content in the Research Portal that you believe breaches copyright or violates any law, please contact openaccess@qub.ac.uk. 

survival: a simulation study

4 Therese M-L Andersson*1, Tor Åge Myklebust ${ }^{2,3}$, Mark J Rutherford ${ }^{4,5}$, Bjørn Møller², Isabelle $5 \quad$ Soerjomataram ${ }^{5}$, Melina Arnold ${ }^{5}$, Freddie Bray ${ }^{5}$, D Maxwell Parkin ${ }^{5,6}$, Peter Sasieni ${ }^{7}$, Oliver 6 Bucher $^{8}$, Prithwish De ${ }^{9}$, Gerda Engholm ${ }^{10}$, Anna Gavin ${ }^{11}$, Alana Little ${ }^{12}$, Geoff Porter ${ }^{13}$, 7 Agnihotram V Ramanakumar ${ }^{14}$, Nathalie Saint-Jacques ${ }^{15}$, Paul M. Walsh ${ }^{16}$, Ryan R Woods ${ }^{17}$, 8 Paul C Lambert ${ }^{1,4}$

9

10 1. Department of Medical epidemiology and Biostatistics, Karolinska Institutet, Stockholm 11 Sweden.

12 2. Cancer Registry of Norway, Institute of Population-based Cancer Research, Oslo, Norway

13 3. Department of Research and Innovation, Møre and Romsdal Hospital Trust, Ålesund, Norway

14 4. Department of Health Sciences, University of Leicester, Leicester, United Kingdom.

15 5. Cancer Surveillance Section, International Agency for Research on Cancer (IARC/WHO), 16 Lyon, France.

17 6. Nuffield Department of Population Health, University of Oxford, Oxford, United Kingdom 18 7. Faculty of Life Sciences \& Medicine, School of Cancer \& Pharmaceutical Sciences, Guys 19 Cancer Centre, Guys Hospital, King’s College London, London, United Kingdom.

20 8. Department of Epidemiology and Cancer Registry, CancerCare Manitoba, Winnipeg, MB, 21 Canada

22 9. Analytics and Informatics, Ontario Health (Cancer Care Ontario), Toronto, Ontario, Canada 23 10. Surveillance and Pharmacoepidemiology, Danish Cancer Society Research Center, 24 Copenhagen, Denmark 
25 11. Northern Ireland Cancer Registry, Queen's University Belfast, Northern Ireland, United

26 Kingdom.

27 12. Cancer Institute NSW, Alexandria NSW, Australia

28 13. Canadian Partnership Against Cancer, Toronto, Ontario, Canada.

29 14. Research-Institute, McGill University Health Center, Montreal, Quebec, Canada

30 15. Nova Scotia Health Authority Cancer Care Program, Registry \& Analytics, Halifax, Nova

31 Scotia, Canada

32 16. National Cancer Registry Ireland, Cork, Ireland

33 17. Cancer Control Research, BC Cancer, Vancouver, British Columbia, Canada

34

35 Corresponding author: Therese M-L Andersson. E-mail: therese.m-l.andersson@ki.se

36

37 Key words: Cancer registry; Death certificate initiated cases; Survival; Simulation study

38

39 Word count: Abstract 248, Main text 3308, Highlights 65.

40

41 Abbreviations: DCN - death certificate notified; DCI - death certificate initiated; DCO - death

42 certificate only; ICBP - International Cancer Benchmarking Partnership; RR - relative risk; RSR

43 - relative survival ratio

44 
Highlights:

- This simulation study shows that including cases initiated through death certificates in the survival analysis of population-based registry data will downwardly bias relative survival estimates.

- Excluding cases initiated through death certificates will in most situations overestimate survival.

- The extent of the bias depends on how missed cases differ from those registered through other routine sources.

- Registries should report the DCI proportion alongside the DCO proportion. 


\section{$46 \quad$ Abstract}

47 Background: Population-based cancer registries strive to cover all cancer cases diagnosed within

48 the population, but some cases will always be missed and no register is $100 \%$ complete. Many

49 cancer registries use death certificates to identify additional cases not captured through other

50 routine sources, to hopefully add a large proportion of the missed cases. Cases notified through

51 this route, who would not have been captured without death certificate information, are referred

52 to as death certificate initiated (DCI) cases. Inclusion of DCI cases in cancer registries increases

53 completeness and is important for estimating cancer incidence. However, inclusion of DCI cases

54 will generally lead to biased estimates of cancer survival, but the same is often also true if

55 excluding DCI cases. Missed cases are probably not a random sample of all cancer cases, but

56 rather cases with poor prognosis. Further, DCI cases have poorer prognosis than missed cases in

57 general, since they have all died with cancer mentioned on the death certificates.

58 Methods: We performed a simulation study to estimate the impact of including or excluding DCI

59 cases on cancer survival estimates, under different scenarios.

60 Results: We demonstrated that including DCI cases underestimates survival. The exclusion of

61 DCI cases gives unbiased survival estimates if missed cases are a random sample of all cancer

62 cases, while survival is overestimated if these have poorer prognosis.

63 Conclusion: In our most extreme scenarios, with $25 \%$ of cases initially missed, the usual practice

64 of including DCI cases underestimated 5-year survival by at most 3 percentage points. 


\section{Introduction}

67 Cancer survival, when estimated from population-based cancer registry data, is an important

68 measure of the overall effectiveness of health systems given it estimates the average prognosis of

69 cancer patients in the entire population. When comparing population-based cancer survival

70 estimates between countries or jurisdictions, there has been some debate on how differences in

71 registration processes and practices affect the observed survival differences (1). Previous studies

72 have investigated different aspects, including the impact of: i) a failure to link cancer cases to

73 their death information; ii) missing long-term survivors; iii) cancer cases notified from death

74 certificates and iv) finding a date of recurrence instead of a date of diagnosis (2-5).

76 In this paper we focus on the impact on estimated survival of including or excluding cases

77 notified through death certificates. Many cancer registries periodically receive notifications of

78 cancer diagnoses based on death certificates, usually denoted as death certificate notified (DCN)

79 cases $(6,7)$. For a majority of these DCN cases, the registry will also receive a notification from

80 another source (e.g. pathology or hospital records). Yet for some cases, no additional

81 notifications will be received, indicating these cases would not have been known to the registry

82 were it not for the use of death certificate information. These cases are therefore not reported to

83 the cancer registry when diagnosed.

85 For the DCN cases with no other notification to the registry, trace-back is often performed to 86 actively ascertain when the cancer was first diagnosed and to verify that the case was a reportable

87 cancer. The subset of DCN cases deemed reportable are referred to as DCI (death certificate 88 initiated) cases $(6,7)$. DCI cases are therefore cases that are included in the cancer register solely 
89 due to the use of death certificate notification, and would not have been reported from another

90 source. DCI cases can be further subdivided into cases where trace-back was successful in

91 finding a date of diagnosis and cases where the trace-back did not yield any additional

92 information. The latter cases are commonly referred to as death certificate only (DCO) cases, and

93 they are a subset of the DCI cases $(6,7)$. Some registries receive death certificate information

94 more rapidly than notifications through other routine sources and therefore have a large group of

95 cases initially notified from death certificates. However, these cases should not be referred to as

96 DCI cases since they are reported to the registry through independent routine sources although at

97 a later time. Only cases that would not have been known to the registry, if it was not for the death

98 certificate, are DCI cases.

99

100 While it is important for cancer registries to include DCI cases to increase the completeness of cancer incidence statistics, including DCI cases when estimating survival will generally lead to

102 biased results. The existence of DCI cases indicates that there are cases in the population who are not notified to the registry through the course of their disease and who are either alive, or have

104 died without cancer mentioned as a cause of death. This is illustrated in Figure 1, the interest is in 105 the survival of all cancer cases, i.e. the yellow box. However, some cancer cases are not 106 registered through routine sources, and missed by the registry at diagnosis, represented by the 107 grey solid box in Figure 1. A cancer registry that does not perform trace-back only includes the 108 cases in the green solid box, those that are registered through routine sources. Some of these 109 individuals will be alive at the time the cancer registry performs the survival analysis, some will 110 have died with cancer mentioned on the death certificate and some will have died due to other 111 causes, but all these cases are included. In the unlikely situation that these cases are a random 112 sample of those in the yellow box this should yield unbiased estimates of survival. When a cancer 
113 registry receives DCN cases, performs trace-back and then include the DCI cases, a subset of the

114 missed cases are also included (the box with light green borders), the subset who died due to

115 cancer (or where cancer is mentioned on the death certificate). The cases missed (not notified

116 through routine sources, the solid grey box) that are still alive or died without cancer mentioned

117 on the death certificate will not be retrieved, and continue to be missed by the registry. Since the

118 DCI cases are not a random sample of the cases missed (solid grey box) by the registry, the

119 inclusion of DCI cases when estimating survival can give biased results, even if the whole group

120 of missed cases are a random sample of all cancer cases. The problem can be illustrated in a

121 simple way by considering all cause survival among 1000 individuals. If the survival probability

122 at 5 years is 0.8 and there is no censoring, one would expect there to be (800 people alive at 5-

123 years $(800 / 1000=0.8)$. If $20 \%$ of cases were initially missed (at random) then there would be 800

124 individuals initially with $800 * 0.8=640$ alive at 5 years $(640 / 800=0.8)$. Of those missed, one

125 would expect $200 * 0.2=40$ to die. Including these in the analysis leads to a 5-year survival of

$126(640 /(800+40)=76.2 \%$, i.e. an underestimate as we have only added individuals to the

127 denominator. There is often concern with respect to the validity of data from those registries

128 unable to use death certificates to find additional cases, since it is known that excluding DCI

129 cases will usually overestimate survival. However, the converse - that including DCI cases almost

130 always underestimates survival is often not recognised.

131

132 The International Cancer Benchmarking Partnership (ICBP) SURVMARK-2 study aims to

133 quantify disparities in cancer survival across high-income countries and identify possible reasons

134 for them. As part of this international partnership, we performed a simulation study using a range

135 of scenarios to quantify the impact on estimated cancer survival of including or excluding DCI 
cases. The overarching aim was to comprehensively understand the potential impact of this bias

137 on benchmarking cancer survival across populations.

\section{Methods}

140 To investigate the impact of including or excluding DCI cases on survival estimates, we

141 simulated cohorts of 5000 cancer patients. For each cancer patient, a time of death due to cancer

142 and a time of death due to other causes was simulated (8), and for each individual, their cause of

143 death was determined by the event that occurred first: either death due to cancer, or death due to

144 other causes. All survival times were censored at 10 years. We used three separate Weibull

145 distributions for simulating time to death, representing a cancer site associated with low (Weibull

146 parameter $\lambda=0.61$ and $\gamma=0.63)$, medium $(\lambda=0.4$ and $\gamma=0.6)$ and high $(\lambda=0.12$ and $\gamma=0.64)$ cancer-

147 specific survival, since the bias we wish to investigate can depend on the underlying cancer

148 survival. We also used two levels (high and low, roughly corresponding to the survival of a 65

149 and an 80 year old in UK) of other cause (expected) survival, also with Weibull distributions

$150(\lambda=0.034 ; \gamma=1.25$ and $\lambda=0.13 ; \gamma=1.19$, respectively $)$, since this can have an additional impact on

151 the bias. The survival and hazard functions for both cancer-specific and other cause survival are

152 shown in the Appendix Figure A1.

$154 \quad 2.1$ Simulating randomly missed cases

155 We simulated the proportion of the cancer cases who were missed, i.e. not notified to the registry, 156 except possibly from death certificates, first assuming that these were a random sample of all

157 cases. Three levels of missingness were investigated: 5\%, 15\% and 25\%. This gave a total of 18

158 simulated scenarios: 3 levels of cause-specific survival, 2 levels of other cause survival and 3

159 levels for proportions of cases not reported to the registry, as listed in Table 1. Within each 
simulated scenario, cases who were simulated to be missed by the registry and who died due to

161 cancer within 10 years from diagnosis were classified as DCI cases. For simplicity, we assumed

162 that the trace-back procedure found the correct date of diagnosis for all DCI cases, and hence

163 there were no DCO cases. In actual registry data, DCO cases will exist, and they are usually

164 excluded from survival analysis since their survival time is not known. This might have

165 implications for the extent of bias in our simulations, however the direction of the bias is not

166 altered.

167

$168 \quad 2.2$ Simulating non-randomly missed cases

169 We added another layer to the 18 base scenarios to investigate the impact of including a

170 prognostic factor for death that is related to the extent of missingness. This prognostic factor was

171 represented by a binary variable X (e.g. advanced stage), that affected the time to death due to

172 cancer. The effect of Factor X was assigned a hazard ratio (HR) of 4, meaning that patients with

173 the prognostic factor had a four times higher cancer-specific mortality rate than patients who did

174 not have Factor X. Assuming $25 \%$ of the patients had this prognostic factor, we then simulated

175 the 18 base scenarios as described above, where the probability of being missed differed by

176 Factor $\mathrm{X}$, while keeping the same overall probabilities of being missing. For each of the 18 main

177 scenarios, 4 sub-scenarios (a, b, c and d) were simulated where the probability of being missed

178 differed between those with and without Factor $\mathrm{X}$ with a relative risk (RR) of 1.5, 2, 3 and 5. For

179 example, a RR of 1.5 means that those with Factor X were $50 \%$ more likely to be missed as those

180 without the factor. The probability of being missed with and without Factor X, as represented in

181 each scenario, is presented in Table 2. When simulating the time to death due to cancer in all

182 these scenarios, the value of factor $\mathrm{X}$ for each individual was replaced by the value minus 0.25 ,

183 so that the average hazard rate follows the Weibull distributions described above. 
$185 \quad 2.3$ Estimating bias in cancer survival estimates

186 . We estimated relative survival ratios (RSR) at 1 and 5 years after diagnosis as measures of

187 cancer survival under two situations: (1) all missed cases were excluded from the analysis, thus

188 representing a situation where DCI cases are not included and (2) DCI cases are included. The

189 relative survival was estimated using flexible parametric models (9-11) with 4 degrees of

190 freedom, without inclusion of any covariates, and using the rate as specified from the Weibull

191 distribution used in simulation of time from death due to other causes for the expected mortality.

192 To calculate the bias in the RSR estimates introduced by excluding or including DCI cases, the

193 RSR estimates for situations (1) and (2) were compared with the true cancer specific survival

194 based on the Weibull distributions used for the simulations.. Both the absolute (as percentage

195 points) and relative (percentage) differences were calculated. The proportion of DCI cases was

196 also estimated as the difference in the number of cases included for the two situations, divided by

197 the number of cases included for situation (2). All results presented are averages based on 1000

198 simulations for each scenario.

199

$200 \quad 2.4$ Sensitivity analysis

201 Scenarios with HRs for Factor X of 1.5 and 2 were also simulated, and results from those

202 simulations are provided in the Appendix.

203

204 3. Results

$205 \quad 3.1$ Randomly missed cases

206 When cases who are missed by the registry were a random sample of all cancer cases occurring in 207 the population, unbiased estimates for the RSR were obtained when DCI cases were excluded 
(Figure 2). Including DCI cases however underestimated survival, since the DCI cases are a

209 selection of those missed who have a poorer prognosis. The size of the bias introduced differed

210 across the 18 simulated scenarios, with the most important factor being the proportion of cases

211 missed. When $5 \%$ of cases were missed (scenarios 1-6), the bias was small, less than 0.5

212 percentage points for 1-year survival and 0.6 percentage points for 5 -year survival. When $15 \%$ of

213 cases were missed (scenarios 7-12) the bias in 1-year survival was still lower than 1.5 percentage

214 point, and just above 1.5 percentage points for 5-year survival. The largest bias -2.5 percentage

215 points for 1-year and 2.8 for 5-year survival - occurred when $25 \%$ of cases were not notified 216 (scenarios 13-18).

218 There was no clear trend in the extent of bias in terms of the prognosis of the cancer (low, 219 medium or high survival), or the level of other cause survival. Rather it was the combination of 220 cancer and other cause survival which was important, since the extent of bias depends on the 221 proportion of the missed cases who were added when the DCI cases were included in the 222 analysis. As the bias will also depend on the true RSR, the relative bias is also presented in 223 Figure 2.

226 For the next set of results (Figure 3) we assumed that cases with a poorer prognosis were more 227 likely to be missed. In this analysis, the exclusion of DCI cases led to an overestimation of 228 survival, and for many scenarios this overestimation was greater than the underestimation 229 introduced when DCI cases were included. The bias introduced by either including or excluding 230 DCI cases was largest for the scenarios where $25 \%$ of cases were missed by the registry, 231 suggesting that the proportion of cases missed was the most important driver of potential bias. 
232 When DCI cases were excluded, the gap between the estimated and true survival widened, with 233 an increasing RR of being missed for those with Factor X. The opposite was true when DCI cases

234 were included. The largest bias observed when including DCI cases was an underestimation of 1235 year survival by 2.7 percentage points and 5-year survival by 2.9 percentage points. The largest 236 bias observed when excluding the DCI cases was an overestimation of 1-year survival by 5.9

237 percentage points and 5-year survival by 5.4 percentage points. Again, there was no clear trend in 238 the extent of bias in terms of cancer-specific survival, or other cause survival.

$240 \quad 3.3$ Proportion of Death Certificate Initiated cases

241 The proportions of DCI cases for each simulated scenario are presented in Table 3. The

242 proportion of DCI cases depends on the proportion of missed cases, since it can never be higher 243 than the proportion missed. For any given value of the proportion missed, the proportion of DCI 244 cases decreased with increasing cause-specific survival, as there would be a diminishing number 245 of cases who die from cancer. On the other hand, the proportion of DCI cases was higher for 246 higher other cause survival. This is because a larger proportion of cases will die due to cancer if 247 fewer die due to other causes. Finally, the proportion of DCI cases also increased with increasing 248 RR of being missed for those with Factor X compared to those without Factor X.

$250 \quad 3.4$ Sensitivity analysis

251 For scenarios where the HR for Factor X was changed to 1.5 or 2, the pattern of the results were 252 similar to the scenarios where the HR was 4, however, the bias introduced by excluding DCI 253 cases was smaller with a lower HR (Appendix Figures A2 and A3). The bias introduced by 254 including DCI cases was less affected by the size of the HR for Factor X. 255 


\section{Discussion}

258 Our simulation study shows that performing trace-back to include DCI cases, does not resolve the 259 problem of missing cases biasing survival estimates, and can in certain circumstances lead to an 260 even larger bias than that resulting from excluding DCI cases from the analyses. The inclusion of

261 DCI cases in cancer registries is a necessary procedure to achieve the highest possible

262 completeness in terms of cancer incidence. When estimating survival, the inclusion of DCI cases

263 will underestimate survival, while their exclusion will overestimate survival. The utilization of

264 death certificates as a source for cancer notifications implies that some cancer cases are not 265 reported to the registry when diagnosed, and even if those missed are a random sample of cases, 266 inclusion of the DCI cases will lead to biased survival estimates. Thus, excluding DCI cases 267 when estimating survival will lead to unbiased survival estimates only if those cases not notified represent a random sample of all cancer cases - which is unlikely in most situations- otherwise, 269 survival will be overestimated if the missed cases have more severe disease.

271 In our study we have demonstrated the impact on survival estimates of including and excluding

272 DCI cases. This has consequences for survival benchmarking. For two countries where one 273 includes DCI cases that were successfully traced back and the other does not, both estimates of 274 cancer survival will be biased, but in opposite directions. Even when comparing two populations 275 with the same practice in terms of including or excluding DCI cases, the bias may be of different 276 magnitudes depending on the true proportion missing within each registry, the mechanisms that

277 dictate the degree of missingness and the amount of trace-back. The inclusion of DCI cases could 278 also lead to greater underestimation if the trace-back doesn't find the true date of diagnosis but 279 rather a later date such as that at recurrence, but this was not evaluated in this study. An 
additional issue when investigating trends over calendar time is that there is less opportunity for 281 cases diagnosed (and missed) more recently, to be obtained from death certificates due to their 282 shorter follow-up.

284 All cancer registries participating in ICBP SURVMARK-2 include DCI cases, although the 285 proportion of DCI cases is often unknown. Unfortunately, most cancer registries are not able to 286 retrospectively identify DCI cases in their data as typically this information is superseded when 287 other information relating to time prior to death is retrieved. However, for registries within ICBP 288 SURVMARK-2 where the proportion of DCI cases is available, a proportion of about $15 \%$ can 289 be observed for cancer sites with poor prognosis, indicating that scenarios 7 and 8 are plausible 290 scenarios for a poor prognosis cancer. For cancer sites with better prognosis, a proportion of DCI 291 cases of about 3-4\% has been observed in real data, indicating that scenarios 3-6 are plausible.

292 However, given the small number of registries that have information on DCI cases, and the 293 uncertainty in the proportion of missed cases, we explored a wider range of scenarios in this 294 study.

296 A few limitations should be noted in relation to our study. We did not simulate an age 297 distribution within the data, but rather investigated two levels of other cause survival. In all 298 simulations we assumed that the prognostic Factor X was only associated with cancer-specific 299 survival, but not other cause survival, which might be violated if the prognostic factor is, for 300 example, the presence of comorbidity. We also assumed that cause of death is recorded accurately for all cases. Another aspect that could be of interest is specification of DCO cases. In our simulations we assumed that the true date of diagnosis is found for all DCI cases, resulting in no DCO cases. We also assumed that all death certificates had been retrieved by the registry by 
304 the time the survival analysis was performed, so all cases were correctly classified. Even so, this

305 simulation study showed clearly how the inclusion of DCI cases underestimates survival, and 306 excluding DCI cases instead overestimates survival if cases who were not notified were not a 307 random sample of all cancer patients in the population.

308

309 The extent of bias largely depends on the proportion of cases who are not notified, but the bias 310 also differs depending on the extent to which the missed cases are notified as DCI cases (i.e. the 311 proportion of the missed cases who have died and had cancer mentioned on their death 312 certificates). It is reassuring to see that our scenarios give a bias of at most 3 percentage points in 313 the situation when DCI cases are included. It is by definition impossible to know the true 314 proportion of cases missed by a registry, but the proportion of DCI cases serves as an important indicator in this respect. Registries should therefore report the proportion of DCI cases along with 316 the more commonly reported proportion of DCO cases.

318 Conflict of interest

319 The authors declare no competing interests.

$321 \quad$ Funding

322 The ICBP is funded by the Canadian Partnership Against Cancer; Cancer Council Victoria;

323 Cancer Institute New South Wales; Cancer Research UK; Danish Cancer Society; National

324 Cancer Registry Ireland; The Cancer Society of New Zealand; NHS England; Norwegian Cancer

325 Society; Public Health Agency Northern Ireland on behalf of the Northern Ireland Cancer

326 Registry; DG Health and Social Care, Scottish Government; Western Australia Department of

327 Health; Public Health Wales NHS Trust. 
329 Acknowledgements

330 The authors would also like to thank Lucie Hooper, Samantha Harrison, Charles Norell, Shanta

331 Keshwala and Charlotte Lynch of Cancer Research UK for managing the programme. The ICBP

332 Clinical Committees for their advice. The ICBP SurvMark-2 Academic Reference Group for

333 providing independent peer review and advice for the study protocol and analysis plan

334 development. Finally, we are thankful to the ICBP Programme Board for their oversight and

335 direction.

336

337 Author statement

338 Where authors are identified as personnel of the International Agency for Research on

339 Cancer/WHO, the authors alone are responsible for the views expressed in this article and they do

340 not necessarily represent the decisions, policy or views of the International Agency for Research

341 on Cancer/WHO.

342 


\section{References}

345 1. Beral V, Peto R. UK cancer survival statistics. Bmj. 2010;341:c4112.

3462 2. Robinson D, Sankila R, Hakulinen T, Moller H. Interpreting international comparisons of cancer 347 survival: the effects of incomplete registration and the presence of death certificate only cases on 348 survival estimates. Eur J Cancer. 2007;43(5):909-13.

$3493 . \quad$ Moller H, Richards S, Hanchett N, Riaz SP, Luchtenborg M, Holmberg L, et al. Completeness of 350 case ascertainment and survival time error in English cancer registries: impact on 1-year survival 351 estimates. Br J Cancer. 2011;105(1):170-6.

352 4. Woods LM, Coleman MP, Lawrence G, Rashbass J, Berrino F, Rachet B. Evidence against the 353 proposition that "UK cancer survival statistics are misleading": simulation study with National Cancer 354 Registry data. Bmj. 2011;342:d3399.

355 5. Rutherford MJ, Moller H, Lambert PC. A comprehensive assessment of the impact of errors in the 356 cancer registration process on 1- and 5-year relative survival estimates. Br J Cancer. 2013;108(3):691-8.

357 6. Parkin DM, Bray F. Evaluation of data quality in the cancer registry: principles and methods Part 358 II. Completeness. Eur J Cancer. 2009;45(5):756-64.

359 7. Bray F, Parkin DM. Evaluation of data quality in the cancer registry: principles and methods. Part 360 I: comparability, validity and timeliness. Eur J Cancer. 2009;45(5):747-55.

3618 8. Crowther MJ, Lambert PC. Simulating biologically plausible complex survival data. Stat Med. 362 2013;32(23):4118-34.

363 9. Royston P, Parmar MK. Flexible parametric proportional-hazards and proportional-odds models 364 for censored survival data, with application to prognostic modelling and estimation of treatment effects. 365 Stat Med. 2002;21(15):2175-97.

366 10. Nelson CP, Lambert PC, Squire IB, Jones DR. Flexible parametric models for relative survival, with 367 application in coronary heart disease. Stat Med. 2007;26(30):5486-98.

368 11. Lambert PC, Royston P. Further development of flexible parametric models for survival analysis. 369 Stata J. 2009;9(2):265-90. 
372 Table 1. Combinations of probability of cases being missed in the registry, cancer-specific

373 survival, and other cause (non-cancer) survival included in the 18 simulated main scenarios.

\begin{tabular}{|c|c|c|c|}
\hline Scenario & Probability missed & $\begin{array}{l}\text { Cancer-specific } \\
\text { survival }\end{array}$ & Other cause survival \\
\hline 1 & 0.05 & Low & Low \\
\hline 2 & 0.05 & Low & High \\
\hline 3 & 0.05 & Medium & Low \\
\hline 4 & 0.05 & Medium & High \\
\hline 5 & 0.05 & High & Low \\
\hline 6 & 0.05 & High & High \\
\hline 7 & 0.15 & Low & Low \\
\hline 8 & 0.15 & Low & High \\
\hline 9 & 0.15 & Medium & Low \\
\hline 10 & 0.15 & Medium & High \\
\hline 11 & 0.15 & High & Low \\
\hline 12 & 0.15 & High & High \\
\hline 13 & 0.25 & Low & Low \\
\hline 14 & 0.25 & Low & High \\
\hline 15 & 0.25 & Medium & Low \\
\hline 16 & 0.25 & Medium & High \\
\hline 17 & 0.25 & High & Low \\
\hline 18 & 0.25 & High & High \\
\hline
\end{tabular}


375 Table 2. Probability of a case with and without prognostic Factor $\mathrm{X}$ being missed by the registry,

376 in four sub-scenarios ${ }^{*}$ for each of the 18 base scenarios.

\begin{tabular}{|c|c|c|c|}
\hline Scenarios & Sub-scenario & $\begin{array}{l}\text { Probability missed } \\
\text { among cases without } \\
\text { Factor X }\end{array}$ & $\begin{array}{c}\text { Probability missed } \\
\text { among cases with Factor } \mathrm{X}\end{array}$ \\
\hline $1-6$ & $\mathrm{a}$ & 0.044 & 0.066 \\
\hline $1-6$ & $\mathrm{~b}$ & 0.040 & 0.080 \\
\hline $1-6$ & $\mathrm{c}$ & 0.033 & 0.100 \\
\hline $1-6$ & $\mathrm{~d}$ & 0.025 & 0.125 \\
\hline $7-12$ & $\mathrm{a}$ & 0.133 & 0.200 \\
\hline $7-12$ & $\mathrm{~b}$ & 0.120 & 0.240 \\
\hline $7-12$ & $\mathrm{c}$ & 0.100 & 0.300 \\
\hline $7-12$ & $\mathrm{~d}$ & 0.075 & 0.375 \\
\hline $13-18$ & $\mathrm{a}$ & 0.222 & 0.333 \\
\hline $13-18$ & $\mathrm{~b}$ & 0.200 & 0.400 \\
\hline $13-18$ & $\mathrm{c}$ & 0.166 & 0.500 \\
\hline $13-18$ & $\mathrm{~d}$ & 0.125 & 0.625 \\
\hline
\end{tabular}

$377{ }^{*}$ Sub-scenarios a to $d$ represent relative risk of being missed in the registry of $1.5 ; 2 ; 3$ and 5 ,

378 respectively 
379 Table 3. Proportion of Death Certificate Initiated (DCI) cases in each simulated scenario.

\begin{tabular}{|l|l||l|l||l|l|}
\hline Scenario & \% DCI & Scenario & \% DCI & Scenario & $\%$ DCI \\
\hline \hline 1 & 3.7 & 7 & 11.3 & 13 & 19.4 \\
$1 \mathrm{a}$ & 3.7 & $7 \mathrm{a}$ & 11.3 & $13 \mathrm{a}$ & 19.4 \\
$1 \mathrm{~b}$ & 3.8 & $7 \mathrm{~b}$ & 11.6 & $13 \mathrm{~b}$ & 19.8 \\
$1 \mathrm{c}$ & 3.9 & $7 \mathrm{c}$ & 12.0 & $13 \mathrm{c}$ & 20.5 \\
$1 \mathrm{~d}$ & 4.1 & $7 \mathrm{~d}$ & 12.5 & $13 \mathrm{~d}$ & 21.3 \\
\hline 2 & 4.4 & 8 & 13.4 & 14 & 22.6 \\
$2 \mathrm{a}$ & 4.3 & $8 \mathrm{a}$ & 13.2 & $14 \mathrm{a}$ & 22.3 \\
$2 \mathrm{~b}$ & 4.4 & $8 \mathrm{~b}$ & 13.3 & $14 \mathrm{~b}$ & 22.5 \\
$2 \mathrm{c}$ & 4.5 & $8 \mathrm{c}$ & 13.6 & $14 \mathrm{c}$ & 22.9 \\
$2 \mathrm{~d}$ & 4.6 & $8 \mathrm{~d}$ & 13.8 & $14 \mathrm{~d}$ & 23.3 \\
\hline 3 & 3.0 & 9 & 9.3 & 15 & 16.2 \\
$3 \mathrm{a}$ & 3.1 & $9 \mathrm{a}$ & 9.6 & $15 \mathrm{a}$ & 16.7 \\
$3 \mathrm{~b}$ & 3.2 & $9 \mathrm{~b}$ & 10.0 & $15 \mathrm{~b}$ & 17.3 \\
$3 \mathrm{c}$ & 3.4 & $9 \mathrm{c}$ & 10.6 & $15 \mathrm{c}$ & 18.2 \\
$3 \mathrm{~d}$ & 3.6 & $9 \mathrm{~d}$ & 11.2 & $15 \mathrm{~d}$ & 19.3 \\
\hline 4 & 3.9 & 10 & 11.9 & 16 & 20.3 \\
$4 \mathrm{a}$ & 3.8 & $10 \mathrm{a}$ & 11.8 & $16 \mathrm{a}$ & 20.2 \\
$4 \mathrm{~b}$ & 3.9 & $10 \mathrm{~b}$ & 12.1 & $16 \mathrm{~b}$ & 20.6 \\
$4 \mathrm{c}$ & 4.1 & $10 \mathrm{c}$ & 12.5 & $16 \mathrm{c}$ & 21.2 \\
$4 \mathrm{~d}$ & 4.2 & $10 \mathrm{~d}$ & 13.0 & $16 \mathrm{~d}$ & 21.9 \\
\hline 5 & 1.4 & 11 & 4.4 & 17 & 8.0 \\
$5 \mathrm{a}$ & 1.6 & $11 \mathrm{a}$ & 5.2 & $17 \mathrm{a}$ & 9.4 \\
$5 \mathrm{~b}$ & 1.7 & $11 \mathrm{~b}$ & 5.6 & $17 \mathrm{~b}$ & 10.1 \\
$5 \mathrm{c}$ & 1.9 & $11 \mathrm{c}$ & 6.1 & $17 \mathrm{c}$ & 11.0 \\
$5 \mathrm{~d}$ & 2.1 & $11 \mathrm{~d}$ & 6.8 & $17 \mathrm{~d}$ & 12.2 \\
\hline 6 & 2.2 & $12 \mathrm{a}$ & 7.0 & 18 & 12.4 \\
$6 \mathrm{a}$ & 2.4 & $12 \mathrm{c}$ & 8.6 & $18 \mathrm{a}$ & 13.5 \\
$6 \mathrm{~b}$ & 2.5 & $12 \mathrm{~d}$ & 9.6 & $18 \mathrm{~b}$ & 14.2 \\
$6 \mathrm{c}$ & 2.7 & 9.4 & $18 \mathrm{c}$ & 16.4 \\
$6 \mathrm{~d}$ & 3.0 & & & & \\
\hline & & 12 & & \\
\hline
\end{tabular}


382 Figure 1. Illustration of Death Certificate Initiated (DCI) cases as a subset of all cases of cancer 383 arising in the population.

384

385 
386 Figure 2. Absolute and relative differences in 1- and 5-year relative survival ratios (RSR) for the 38718 base scenarios $^{*}$ described in Table 1 (where the missed cases are a random sample of all 388 cases): including or excluding death certificate initiated cases compared to the full cohort.

389 Negative values refer to underestimation of survival, and positive values overestimation of 390 survival.

391

392

393 Note that the absolute and relative differences are shown with different scales 394

$395{ }^{*} 5 \%, 15 \%, 25 \%$ missing registration for scenarios 1-6, 7-12 and 13-18 respectively with different combinations of 396 low, medium and high cancer specific survival and level of other cause survival. 
398 Figure 3. Absolute and relative differences in 1- and 5-year relative survival ratios (RSR) for the 39972 simulation scenarios ${ }^{*}$ described in Table 1 and Table 2: including or excluding death 400 certificate initiated cases compared to the full cohort. For each of the 18 base scenarios, sub401 scenario a to d are displayed with varying degrees of transparency, a with least and d with most 402 transparent circles. Negative values refer to underestimation of survival, and positive values 403 overestimation of survival.

404

405

$406 \quad$ Note that the absolute and relative differences are shown with different scales

407

$408{ }^{*} 5 \%, 15 \%, 25 \%$ missing registration for scenarios 1-6, 7-12 and 13-18 respectively with different combinations of 409 low, medium and high cancer specific survival and level of other cause survival. Sub scenarios a-d: Relative risk of 410 being missed for those with Factor X (with higher risk of dying) relative to those without of $1.5,2,3$ and 5 , 411 respectively. 


\section{Appendix}

414 Figure A1. Cause-specific and other cause survival (a) and hazard (b) functions used in

415 simulations, representing scenarios with low, medium and high cancer-specific survival and high

416 and low other cause survival.

417

418

419 (a)

420

421

422

(b)

423 
424 Figure A2: Absolute and relative differences in 1- and 5-year relative survival ratios (RSR) for 425 the 72 simulation scenarios ${ }^{*}$ described in Table 1 and Table 2 using hazard ratio of 1.5 for cases 426 with the prognostic Factor X.

427

428

429 Note that the absolute and relative differences are shown with different scales

430

$431 * 5 \%, 10 \%, 15 \%$ missing registration for scenarios 1-6, 7-12, 13-18 respectively with different combinations of low, 432 medium and high cancer specific survival and level of other cause survival. Sub scenarios a-d: Relative risk of being 433 missed for those with Factor X (with higher risk of dying) relative to those without of 1.5, 2, 3 and 5, respectively. 434 
435 Figure A3. Absolute and relative differences in 1- and 5-year relative survival ratios (RSR) for 436 the 72 simulation scenarios ${ }^{*}$ described in Table 1 and Table 2 using hazard ratio of 2 for cases 437 with the prognostic Factor X.

438

439

440 Note that the absolute and relative differences are shown with different scales

441

$442{ }^{*} 5 \%, 15 \%, 25 \%$ missing registration for scenarios 1-6, 7-12, 13-18 respectively with different combinations of low, 443 medium and high cancer specific survival and level of other cause survival. Sub scenarios a-d: Relative risk of being 444 missed for those with Factor X (with higher risk of dying) relative to those without of $1.5,2,3$ and 5 , respectively. 445 